\title{
Purulent penile ulcers of patients in Singapore
}

\author{
T THIRUMOORTHY,* E H SNG, $\uparrow$ S DORAISINGHAM, $\uparrow$ A E LING, $\uparrow$ K B LIM,* AND C T LEE* \\ From the*Middle Road Hospital and the $\dagger$ Department of Pathology, Singapore General Hospital, Singapore
}

SUMMARY In 80 patients with painful purulent penile ulcers who attended the outpatient service of Middle Road Hospital in Singapore, Haemophilus ducreyi was isolated from 18 (22\%), herpes simplex virus type 2 from nine (11\%), and Neisseria gonorrhoeae from eight (10\%). Primary pathogens were not isolated from $45(57 \%)$ men. Painful purulent penile ulcers were more common in uncircumcised men, and patients had often acquired the disease after sexual intercourse with prostitutes in Singapore.

\section{Introduction}

Purulent painful penile ulcers are a common problem in patients who attend sexually transmitted diseases (STD) clinics in the tropics. Though the ulcers are often typical of clinical chancroid, the clinical impression may be erroneous. ${ }^{1}$ Moreover the simultaneous occurrence of two or more pathogens in the same ulcer has been described. ${ }^{2}$ A previous local study of genital ulcers in Singapore confirmed the polymicrobial aetiology of penile ulcers, though isolation of Haemophilus ducreyi was not successful on the solid medium used. ${ }^{3}$

In the study published here we studied mainly purulent painful penile ulcers, which we would clinically call "chancroidal ulcers", in an attempt to define the aetiological agents and clinical and epidemiological features.

\section{Patients and Methods}

Though we studied 100 patients with clinical diagnoses of "chancroidal ulcers" who attended the outpatient service of Middle Road Hospital, (the main STD clinic in Singapore), adequate data were available from only 80. Men who had received antibiotics less than a week before were excluded from the study.

A brief sexual history was taken and clinical

Address for reprints: Dr C T Lee, Middle Koad Hospital, 250 Middle Road, Singapore 0718, Republic of Singapore

Accepted for publication 12 November 1985 examination carried out. Five specimens were taken from the largest ulcer for culture for $\boldsymbol{H}$ ducreyi, Neisseria gonorrhoeae, other aerobes, chlamydiae, and herpes simplex virus (HSV). Dark ground examination for spirochaetes was undertaken on exudate from the ulcer. Blood samples for serological tests for syphilis (Venereal Disease Research Laboratory (VDRL) and fluorescent treponemal antibody absorbed (FTA-ABS) tests) and antibodies to HSV were taken on entry into the study. Further blood samples for testing for antibodies to HSV were taken two weeks later, and the VDRL and FTA-ABS test were repeated six weeks later.

\section{LABORATORY METHODS}

For the cultivation of aerobic organisms, $N$ gonorrhoeae, and $H$ ducreyi three successive specimens were directly inoculated on to blood nutrient agar plates, modified Thayer-Martin medium, and chancroid medium. The chancroid medium was made from Columbia agar (Oxoid), fetal calf serum 5\%, human packed erythrocytes $5 \%$, IsoVitalex $1 \%$, and vancomycin $3 \mathrm{mg} / 1$. After inoculation, all the plates were placed in a moistened candle extinction tin and incubated at $35^{\circ} \mathrm{C}$.

The plates were examined after 48 hours. $N$ gonorrhoeae was identified by colonial morphology, oxidase reaction, and Gram stained appearance on microscopy. $\boldsymbol{H}$ ducreyi was identified by the characteristic colonial morphology and microscopy. Biochemical tests were carried out on selected isolates. Culture plates for $\boldsymbol{H}$ ducreyi were examined thereafter daily until the fifth day, and were then discarded. 
Calgiswabs were used to collect the specimens for $C$ trachomatis isolation. Each specimen was placed in sucrose phosphate buffer and transported in ice to the laboratory. Cycloheximide treated McCoy cells were used to cultivate the organism. After incubation for 48 and 72 hours, the cells were stained with Giemsa and examined under dark field microscopy for fluorescent inclusions.

Specimens for HSV culture were collected in Hank's virus transport medium which contained penicillin, gentamicin, and amphotericin B to suppress bacterial and fungal growth. They were centrifuged and inoculated within two to three hours of collection, $0 \cdot 3$ $\mathrm{ml}$ of the specimen being inoculated into each of two tubes of human embryo lung fibroblast cell cultures. Tubes were incubated in roller drums at $35^{\circ} \mathrm{C}$ and examined daily for cytopathic effect (CPE). Negative cultures were discarded after 10 days' observation. Positive cultures usually showed CPE on the first day after inoculation, but less often on the second or third day. Cultures showing 2+ CPE or more were scraped off the tubes, fixed on to glass microscope slides, and stained by the direct immunofluorescent method with monoclonal antibodies to HSV types 1 and 2. These reagents were purchased from Biomerieux (Marcy l'Etoile, Charbonnières les Bains, France).

Serum samples were tested for antibody to HSV by the complement fixation test. Patients were considered to have a positive result if there was a fourfold or greater rise in antibody titre between pairs, or if there was a titre of $1 / 128$ or more in one of the pairs.

\section{Results}

\section{EPIDEMIOLOGY}

Of the 80 men (mean age 23 , range 17 to 45 years), 68 $(85 \%)$ were Chinese, $11(13 \cdot 8 \%)$ Indian, and 1 (1.3\%) Malay. Of all patients attending the Middle Road Hospital, $75 \%$ were Chinese, $10 \%$ Indian, and $15 \%$ Malay.

Female prostitutes were cited by $70(88 \%)$ patients as their source (primary) sexual contact, four (5\%) named casual sexual contacts, four (5\%) regular girl friends, and two (2\%) wives. Sixty five (81\%) patients acquired the disease in Singapore, nine $(11 \%)$ in Taiwan, and six (8\%) in Thailand. The mean incubation period was six (range one to 35 ) days.

\section{CLINICAL FEATURES}

Fifty four $(68 \%)$ of the patients had fewer than three ulcers and 17 (21\%) had four to six. The mean diameter of the ulcers was $7 \mathrm{~mm}$ (range $2 \mathrm{~mm}$ to 30 $\mathrm{mm}$ ), and $42 \%$ of them were exquisitely tender. Thirty (38\%) patients had bilateral and $29(36 \%)$ unilateral inguinal lymphadenopathy; 21 (26\%) and no lymph node enlargement. The glands were extremely tender in only seven (12\%) of the 59 patients with lymphad- enopathy.

\section{AETIOLOGICAL AGENTS}

The table shows that $H$ ducreyi was isolated from 18 (23\%), HSV from nine (11\%), and $N$ gonorrhoeae from five $(6 \%)$ men, and in three patients $H$ ducreyi and $N$ gonorrhoeae were isolated from the same ulcer. Early infectious syphilis was detected by serology in two patients, one of whom had primary syphilis. All dark ground examinations gave negative results. Chlamydia trachomatis was not isolated from any of the purulent penile ulcers.

TABLE Aetiological agents isolated from 80 men attending Middle Road Hospital, Singapore, with purulent penile ulcers, 1984.

\begin{tabular}{lr}
\hline Primary pathogens isolated & No (\%) \\
\hline Haemophilus ducreyi only & $18(23)$ \\
Herpes simplex virus type 2 only & $9(11)$ \\
Neisseria gonorrhoeae only & $5(6)$ \\
$H$ ducreyi and $N$ gonorrhoeae & $3(4)$ \\
Treponema pallidium (serology only) & $2(3)$ \\
None & $43(54)$ \\
\hline
\end{tabular}

No organisms were isolated from $24(30 \%)$ patients, and only aerobes, including staphylococci, streptococci, coliforms, and enterococci, were isolated from a further $19(24 \%)$ patients. As these aerobes were considered to be secondary invaders we concluded that in $43(54 \%)$ patients no primary pathogens were isolated. These aerobic organisms were also isolated from eight patients with $H$ ducreyi, four with HSV and two with gonococcal ulcers.

\section{$H$ ducreyi}

$H$ ducrey $i$ was isolated from the ulcers of 18 patients, and in seven men it was the only organism isolated. Three yielded both $H$ ducreyi and $N$ gonorrhoeae and the remaining eight patients had additional aerobic infections.

The mean age of patients yielding $H$ ducrey $i$ was 22 years, and all were uncircumcised. The mean incubation period was seven (range one to 35 ) days. With one exception, the disease had been acquired from female prostitutes; 12 in Singapore, four in Taiwan, and one in Indonesia.

The mean number of ulcers was three (range one to eight), but nine of the 18 patients presented with a single ulcer. Eight ulcers were exquisitely painful. Seven (39\%)patients yielding $H$ ducrey $i$ had inguinal lymphadenopathy (five unilateral, two bilateral) and in only one with unilateral enlargement were the glands severely tender. Bubo formation was not noted.

Herpes simplex virus (HSV)

HSV was isolated from nine patients; each isolate was of type 2. A fourfold rise of antibody titre was noted in six patients, two of whom had positive cultures. Five 
patients had HSV antibody titres of $1 / 128$ or more. Considering the isolation and serological results together, 18 (23\%) patients were diagnosed as having HSV infection. As a high background level of antibodies was found in this study population, titres above $1 / 128$ may not correlate with recent infection.

In the nine patients from whom HSV type 2 was isolated the mean age was 22.6 years. Six of these nine men acquired the infection in Singapore and one each in Malaysia, Thailand, and Taiwan. Six of the nine patients cited prostitutes as the source of infection, two casual sexual contacts, and one a regular girl friend.

The mean incubation period was three days (range one to six days). Seven men had fewer than four ulcers, and two presented with a single ulcer. Only one ulcer was described as being severely painful. Lymph nodes were enlarged in six patients (four had bilateral and two unilateral enlargement) and were mildly to moderately tender.

\section{$N$ gonorrhoeae}

$N$ gonorrhoeae was isolated from five ulcers. Both $N$ gonorrhoeae and $H$ ducreyi were isolated from three patients. Four isolates were penicillinase producing. Seven men had acquired the disease from prostitutes (six in Singapore and one in Thailand). Only three of these patients had urethral discharge, one man had subpreputial discharge and phimosis.

\section{Discussion}

The polymicrobial nature of purulent penile ulcers has been confirmed in this study. Chancroid is the commonest cause of genital ulcers in Africa, accounting for $62 \%$ of cases in Nairobi ${ }^{4}$ and $42 \%$ in Swaziland. ${ }^{5}$ In the United States of America, however, $H$ ducreyi accounted for $2 \%$ in Detroit ${ }^{2}$ and $1 \%$ in Seattle (Caine VA, unpublished observation). In Thailand ${ }^{6}$ the isolation rate of $H$ ducrey $i$ was $36 \%$ and in Singapore $8 \%{ }^{7}$ In this study $22 \%$ of purulent ulcers were chancroidal. On the other hand, the isolation rates of HSV from genital ulcers were $2 \%$ in $\mathrm{Nairobi}^{4} 11 \%$ in Swaziland, ${ }^{5} 12 \%$ in Thailand, ${ }^{6} 17 \%$ of genital ulcers and $11 \%$ of purulent penile ulcers in Singapore, ${ }^{7} 40 \%$ in Detroit ${ }^{2}$ and $55 \%$ in Seattle (Caine VA, unpublished observation).

These studies show an inverse relation between the isolation of $\boldsymbol{H}$ ducreyi and HSV. This was probably related to socioeconomic development and the accessibility and availability of medical services and antibiotics. In more developed countries the availability of medical facilities and antibiotics has led to a reduction in the incidence of chancroid, thus enabling HSV to fill the vacuum. Another factor contributing to this different pattern of disease is related to the pattern of sexual behaviour. Purulent penile chancroid ulcers are often acquired from the promiscuous middle and lower income prostitutes in developing countries. ${ }^{47}$
The other interesting feature of this study is that it confirms chancroid as a disease that manifests itself most commonly in uncircumcised men. In Singapore the Malays are Muslims by religion and thus are circumcised. In this study of purulent penile ulcers one patient was a Malay, whereas $10 \%$ to $15 \%$ of patients attending the STD clinic were Malays. Of the 18 patients yielding $H$ ducreyi, none were Malays. There was also a low representation of Malays among men with genital warts. Considering that the Malays are living in the same sexual and biological environment as the Chinese and Indians, circumcised men appear to be protected against chancroid ${ }^{4}$ and genital warts. The proportion of Malays with gonorrhoea and syphilis is comparable with the numbers attending the clinic.

This study confirms that genital ulcers caused by HSV can present as purulent painful penile ulcers indistinguishable from chancroid. Bacterial superinfection of genital herpes is also common, even in nonimmunocompromised patients, in contrast to certain popular beliefs. ${ }^{8}$ 'The bacteria implicated include gonococci, coliforms, alpha streptococci, diptheroids, and enterococci. In South East Asia, prostitutes continue to play a pivotal part in the spread of genital infection with HSV.

We think that the $54 \%$ of ulcers from which no primary pathogens were isolated were either cases of chancroid or infected genital herpes. The epidemiological and clinical features were similar in the culture positive and culture negative groups. We were unable to isolate the organisms, possibly because of the fastidious nature of the agents, the long presence of the ulcers, the previous use of antibiotics, or interference of growth in the media by bacterial superinfection.

This study was supported by Roche Far East Research Foundation.

\section{References}

1. Chapel TA. How reliable is the morphological diagnosis of penile ulcerations? Sex Transm Dis 1977;4:150-2.

2. Chapel TA, Brown WJ, Jeffries C, Steward JA. The microbiological flora of penile ulcerations. $J$ Infect $D i s$ 1978;137:50-6

3. Rajan VS, Doraisingham M, Sng EH, Lim AL. Polymicrobial aetiology of genital ulcers. Singapore Med J 1982;23:207-13

4. Nsanze H, Fast MV, D’Costa LJ, Turkei P, Curran J, Ronald A. Genital ulcers in Kenya. British Journal of Venereal Diseases 1981;57:378-81.

5. Meheus A, Van Dyck E, Ursi J P, Ballard RC, Piot P. Etiology of genital ulcerations in Swaziland. Sex Transm Dis 1983;10:30-5.

6. Taylor DN, Duangmani C, Suvongse $C$, et al. The role of Haemophilus ducreyi in penile ulcers in Bangkok, Thailand. Sex Transm Dis 1984;11:148-51

7. Rajan VS, Sng EH, Lim AL. The isolation of $H$ ducreyi in Singapore. Ann Acad Med Singapore 1983;12:57-60

8. Corey L. Genital herpes. In: Holmes KK, Mårdh PA, Sparling PF, Wiesner PJ, eds. Sexually transmitted diseases, New York: McGraw-Hill, 1984:449-740. 